\title{
Juridictionnalisation du droit penitentiaire congolais: défis et perspectives.
}

\author{
Eliezer Costa KOLESHA TSHANGALA*
}

\section{INTRODUCTION}

"En ce monde et dans l'autre, les méchants sont toujours bien embarrassants ». Ces paroles de Jean Jacques Rousseau dans «Les confessions » semblent marquer du sceau de l'éternité$^{1}$. Que faire du délinquant? Cette question, si elle se posait au $19^{\mathrm{e}}$ siècle, semble récurrente encore aujourd'hui. Le débat sur les prisons, leur existence et leur utilité en République Démocratique du Congo est loin d'être clos;

La politique gouvernementale, visant à édifier les établissements pénitentiaires congolais en vue d'améliorer les conditions de détention et désengorger les prisons, est restée lettre morte et n'a pu trouver de solutions favorables pour les détenus congolais. Le système pénitentiaire congolais actuel tend à faire de la peine d'emprisonnement la reine des peines, et a eu, pour conséquence désastreuse, de faire du condamné un laissé-pour-compte (rejeté par tous) et, dès lors que celui-ci est incarcéré, la société se désintéresse de lui et ses droits ne sont que des poussières de droits.

L'exécution des peines privatives de liberté en Droit congolais est la phase pendant laquelle le condamné exécute la peine qui a été prononcée à son encontre par le tribunal. Elle se situe donc en aval de la condamnation. Et, seule l'Administration, et notamment l'Administration pénitentiaire est chargée de l'exécution de cette condamnation en Droit pénitentiaire congolais ${ }^{2}$. Ainsi, la personne détenue est justiciable dont les droits se rapprochent du droit commun. Elle possède un statut juridique supposant l'existence des obligations mais également des droits. Elle bénéficie, au moins en théorie, de moyens juridiques efficaces pour garantir, en détention et devant le juge, la protection de ses droits tout au long de sa détention au sein d'un établissement pénitentiaire.

De la sorte, le droit au respect de la légalité n'est plus contradictoire avec l'emprisonnement et suppose que les personnes privées de liberté bénéficient du droit effectif de requé-

* Eliezer Costa KOLESHA TSHANGALA, Chef de Travaux à l'Université de Lubumbashi et Juge au Tribunal de Grande Instance de Lubumbashi.

1 JEAN JACQUES ROUSSEAU cité par LECOINTE VIRGINIE, La juridictionnalisation de l'exécution des peines, Mémoire d'études approfondies, Ecole doctorale $n^{\circ} 74$, Faculté des sciences juridiques politiques et sociales, Université de Lille II, Droit et Santé, 2001-2002, p.4.

2 Nous pensons à ce sujet par cette étude, créer un courant nouveau qui tend à faire du condamné (détenu) un véritable citoyen, un être sujet des droits à part entière, un individu jouissant de ses droits, c'est-à-dire faire du condamné un citoyen disposant des mêmes droits que toute autre personne à l'exception de la liberté d'aller et de venir. L'idée du condamné-citoyen selon notre vision aboutira de manière incontournable à lui reconnaitre (condamné) ses droits en détention et le condamné devient ainsi l'acteur principal de l'application de sa peine. 
rir. Toutefois, rendre accessibles les moyens de contestation juridictionnels des personnes détenues selon notre entendement, ne signifie pas pour autant qu'ils soient efficaces en pratique. Le contentieux pénitentiaire recèle des spécificités exacerbées par le contexte dans lequel pourraient être mis en œuvre les recours ${ }^{3}$.

La confrontation entre les intérêts, le plus souvent sécuritaires, de l'Administration pénitentiaire et la protection des droits des détenus, contribue à rendre difficilement surmontable ce décalage que le juge ne parvient pas ou ne souhaite pas combler. Celui-ci recherche, avec pragmatisme, l'équilibre pénitentiaire qu'il estime nécessaire au respect juste des intérêts antagonistes. Dès lors, il est possible d'affirmer qu'à la mise à disposition théorique de moyens juridiques de contestation répond le constat d'une pratique peu favorable à l'émergence de droits insusceptibles d'être totalement reconnus, dans leur utilité, par la voie prétorienne. L'objectif originel de la prison est de rééduquer les personnes

détenues, d'abord par les châtiments corporels puis par les sanctions infligées à l'âme. L'enfermement est une mesure de maintien de l'ordre par la neutralisation des personnes dangereuses et leur exclusion de la société. Dès lors, l'objectif est d'enfermer pour punir et de punir pour réinsérer ${ }^{4}$.

Après la révolution française et sous l'influence de la pensée des philosophes des Lumières, l'idéologie humaniste a permis l'émergence de principes fondamentaux. Les principes de liberté, d'égalité, de proportionnalité et de légalité des peines issus de la Déclaration des droits de l'homme de 1948 font désormais partie du socle du Droit pénal ${ }^{5}$. Cependant, l'influence de cet instrument juridique international des Nations Unies reste limitée dans la mesure où ces dispositions ne sont pas contraignantes. L'émergence du droit en prison s'est faite, il y a une quarantaine d'années. Le contrôle juridictionnel a, dans un premier temps, donné lieu à une jurisprudence qui peut être qualifiée de jurisprudence d'opportunité ${ }^{6}$.

La mise en balance des intérêts opposés de l'Administration pénitentiaire et des personnes détenues a conduit le juge à faire évoluer au cas par cas le droit, marquant une certaine frilosité de sa part, mais aussi peut-être, le souhait de ne rien brusquer. La confronta-

3 Il ne doit jamais être permis à l'autorité administrative des prisons de léser illégalement des intérêts des personnes privées de liberté car celles-ci doivent bénéficier du droit de recours effectif et nous préconisons pour cela l'option de toujours se trouver un juge de la légalité de son action.

4 M. LORCY, "L'évolution des conceptions de la peine privative de liberté », in CRDF, 2004, $n^{\circ} 3, p$. 11 .

5 «Nul homme ne peut être accusé, arrêté ou détenu que dans les cas déterminés par la loi et selon les formes qu'elle a

prescrites (...) ». "La loi ne doit établir que des peines strictement et évidemment nécessaires, et nul ne peut être puni qu'en vertu d'une loi établie et promulguée antérieurement au délit, et légalement appliquée ».

6 CE, Ass., 17 février 1995 Marie, req. $n^{\circ}$ 97754, Rec. Lebon, p. 83. Infra. Partie 2, Titre 1, Chapitre 1. 
tion de la sécurité et des droits de l'homme, apprécié par le prisme du droit, a entrainé un décalage entre les principes affirmés par les textes et la pratique ${ }^{7}$.

La juridictionnalisation peut être définie comme le fait de confier à une juridiction le contrôle d'une situation, une juridiction étant elle-même un organe chargé de trancher les litiges au moyen d'une décision appelée jugement. Ce jugement est un acte juridictionnel qui ne se conçoit pas sans une contradiction préalable et doit être motivé. Une fois rendu, ce jugement bénéficie de l'autorité de la chose jugée et ne peut être modifié que dans le cadre strict des voies de recours ${ }^{8}$.

Ainsi, la juridictionnalisation d'un acte consiste donc à donner la qualification d'acte juridictionnel à un acte qui ne l'était pas, que ce soit un acte d'administration judiciaire ou un acte gracieux. La juridictionnalisation se distingue donc de la judiciarisation. En effet, cette dernière consiste à judiciariser un acte, c'est-à-dire à le soumettre à l'autorité judiciaire, alors qu'auparavant, par exemple, il était pris par une autorité administrative. Or la juridictionnalisation, quant à elle, intervient alors même que l'acte était déjà pris par un magistrat mais ne possédait pas la qualité d'acte juridictionnel' ${ }^{9}$.

S'il parait important pour nous de faire la distinction de ces deux notions, il faut tout de même souligner que certains auteurs tendent à les assimiler, tout en reconnaissant leurs différences, ce qui participe donc d'une certaine confusion. La juridictionnalisation de l'exécution de la peine privative de liberté suppose comme préalable que les actes rendus en ce domaine ne sont pas juridictionnels.

L'accès des personnes détenues aux recours signifie l'utilisation possible des moyens juridiques de Droit public et, l'accès au droit en détention à l'utilisation des recours administratifs puis juridictionnels, en passant par les voies juridiques indirectes moins connues. L'accès au droit en détention signifie également que devra être déterminée, l'effectivité de ces recours, et leur efficacité; en d'autres termes, leur capacité à prospérer lorsqu'ils sont utilisés par les personnes détenues.

Il faut d'ores et déjà préciser que la problématique de notre démarche scientifique ne repose pas sur une seule question mais sur un ensemble d'interrogations. Pour mener à bien cette réflexion axée sur la juridictionnalisation du Droit pénitentiaire congolais, il nous a semblé souhaitable de poser une série de questions, qui peuvent être énoncées et regroupées comme suit : quelle est donc la pertinence de la juridictionnalisation de l'exécution des peines privatives de liberté en République Démocratique du Congo, tant au niveau de la théorie que de la pratique?

7 H.BELRHLI-BERNARD, « Le juge administratif et le contrôle de l'administration pénitentiaire », in, J.-C.

FROMENT, M. KALUSZYNSKY, L'administration pénitentiaire face aux principes de la nouvelle gestion publique,PUG, 2011, p. 175-186.

8 FAUCHER, P.; La juridictionnalisation de l'application des peines, une révolution tranquille, RPDP, 2001, p.215.

9 CARTIER M-E, «La Judiciarisation de l'exécution des peines », Rev. Sci. Crim, 2001, p.88. 
Ainsi, différentes d'autres questions, toutes aussi essentielles les unes que les autres se posent à savoir : les personnes détenues en République Démocratique du Congo disposentelles de moyens juridiques prévus par le Droit positif? Ces moyens sont-ils connus et accessibles en détention? Ou alors sont-ils suffisants pour protéger les droits matériels des personnes détenues? Les droits reconnus aux personnes détenues sont-ils simplement déclaratoires ou se transforment-ils en véritables droits subjectifs? Existe-t-il des solutions susceptibles d'améliorer ces moyens juridiques de contestation? Existe-t-il des garanties juridictionnelles pour une meilleure protection effective et efficace des détenus? Enfin, sur le plan des principes : la réforme du système pénitentiaire congolais est-elle nécessaire? Comment fonctionne l'exécution des peines privatives de liberté et quelles en sont les difficultés rencontrées en République Démocratique du Congo?

En guise d'hypothèses à la problématique de notre étude, il apparait que la juridictionnalisation de l'exécution de la peine privative de liberté en République Démocratique du Congo, tant sur les plans théorique que de la pratique, présente un bilan que l'on pourrait qualifier de globalement mitigé compte tenu des difficultés qui viennent assombrir ce tableau. Nous notons par ailleurs que l'effectivité théorique des moyens juridiques de contestation dont disposent les personnes détenues en République Démocratique du Congo répond à une mise en œuvre pratique qui révèle un accès conditionné voire l'inaccessibilité à ces dispositifs et à l'efficacité incertaine.

Ces outils juridiques de contestation sont insusceptibles pour l'heure, d'être totalement rétablis dans leur utilité par le juge congolais. Cette étude pratique est également théorique dans la mesure où elle vise à proposer une transformation de recours pour excès et/ou abus de pouvoir en milieu pénitentiaire congolais, sous l'angle d'une redéfinition de l'office du juge administratif.

Pour y arriver, il va falloir préciser le contour sémantique de concepts qui constituent la charpente de l'étude sous examen, avant de relever les faiblesses de la législation pénitentiaire congolaise quant à l'accès au droit des personnes détenues au recours juridictionnel, dans le but de dégager les pistes de solution susceptibles de contribuer à l'amélioration sur cette question.

\section{A. LA JURIDICTIONNALISATION DU DROIT PENITENTIAIRE CONGOLAIS.}

L'étude des droits procéduraux dans le contentieux carcéral apparaît comme la suite logique de la reconnaissance de droit aux personnes détenues ${ }^{10}$ dans la mesure où les recours tant

10 .L'utilisation que nous ferons des termes détenus, personnes détenues, personnes incarcérées sont synonymes, et doivent s'entendre comme la personne majeure condamnée par la juridiction pénale à une peine privative de liberté en maison d'arrêt ou en établissement pour peines 136. Toutefois, il pourra être fait ponctuellement état de situations relatives aux mineurs détenus 137 ou aux femmes incarcérées 138. Il s'agira alors d'analyser une situation contentieuse susceptible d'éclairer la démonstration, sans qu'elle ne soit biaisée par les différences de statut de ces personnes, également privées de liberté. En d'autres termes, condamnés et prévenus peuvent être confondus lorsque leur statut pénal, différent, ne suffit pas à les distinguer du point de vue du contentieux pénitentiaire. 
administratifs que juridictionnels ne sont qu'un moyen d'obtenir in fine la protection effective des droits matériels. Ainsi, l'intérêt d'une telle étude est vraiment contemporain dans la mesure où l'actualité quotidienne évoque les questions carcérales, qu'il s'agisse des mauvaises conditions de détention, attentatoires à la dignité des personnes détenues, de la question de la surpopulation carcérale et de l'encellulement individuel ou de celle des fouilles à nu. Toutefois, ce constat ne pourra pas empêcher la naissance et le développement du contentieux public pénitentiaire, fruit de l'existence d'un droit pénitentiaire peu respecté, de la détermination d'un ordre juridictionnel compétent et de voies de Droit pour y accéder.

L'acception large du Droit pénitentiaire recouvre deux thèmes. D'une part, la réglementation dans l'univers carcéral et d'autre part, le Droit pénal relatif à la peine. Le premier aspect recouvre les dispositions juridiques permettant aux établissements pénitentiaires et au service public qu'ils gèrent de fonctionner correctement. Ces dispositions encadrent les relations au sein des établissements pénitentiaires, et régissent la vie carcérale. Cette réglementation constitue le Droit public pénitentiaire et relève de la compétence du juge administratif. Le deuxième aspect concerne l'application de la peine dévolue à la compétence du juge répressif et soumise au contrôle du ministère public. Cet aspect judiciaire du Droit pénitentiaire ne sera examiné, de façon sommaire, qu'à l'occasion de l'étude des conséquences juridiques des sanctions disciplinaires sur la durée de la peine ${ }^{11}$.

C'est donc une conception étroite de la notion du Droit pénitentiaire qui sera retenue pour l'étude. Celui-ci peut se définir, selon la Professeure Martine Herzog-Evans, comme « la discipline juridique qui traite de l'individualisation des peines en milieu fermé, du statut et du fonctionnement des établissements pénitentiaires, du statut de leurs personnels, des conditions de détention, des droits et obligations des personnes détenues, du maintien des liens familiaux de ces dernières, du maintien de l'ordre et de la sécurité ainsi que du régime de la contrainte et des sanctions qui s'appliquent aux reclus $»{ }^{12}$.

Il s'agit donc d'un droit «pluriel ou mixte » dans la mesure où il fait appel à la fois au Droit public et au Droit pénal. Mais le Droit pénitentiaire se «distingue du droit de l'application des peines qui constitue le prolongement du Droit pénal et du droit de la peine », et dont la nature est seulement pénale ${ }^{13}$.

Cette distinction peut être difficile à appréhender en pratique. Elle semble toutefois définitivement fixée, malgré le fait qu'une partie de la doctrine pénaliste remette en cause cette démarcation afin de justifier le rattachement de la discipline pénitentiaire à la compétence du juge pénal. Ce transfert de compétence n'est pour l'heure pas envisagé en Droit congolais. La conséquence négative, mais logique, a été de donner naissance à un Droit de

Les prévenus sont également des personnes détenues. Elles sont en attente de jugement ce qui implique qu'elles soient, sous certains aspects, soumis à un régime spécifique.

11 J. PRADEL, « Rapport introductif », in RPDP, 2007, n spécial, p. 7.

12 M. HERZOG-EVANS, Droit Pénitentiaire, 2ème éd. Dalloz, Dalloz action 2012-2013, $n^{\circ} 002.21$, p. 7.

13 E. PECHILLON, Sécurité et droit du service public pénitentiaire, LGDJ 1998, nº 18 p. 14 et n 22 p. 17. 
la détention extrêmement complexe. Un Droit mixte, administratif et pénal, et aux multiples sources, règlementaires, législatives et jurisprudentielles. Le Droit pénitentiaire devient un « patchwork » de normes en perpétuel mouvement, si bien qu'il est difficile de se l'approprier efficacement afin qu'il devienne arme contentieuse ${ }^{14}$.

Il faut noter par ailleurs que la notion de contentieux est propre au Droit public ${ }^{15}$. Elle se matérialise en Droit pénitentiaire par le parcours du détenu requérant, de la demande initiale faite auprès de l'Administration pénitentiaire jusqu'à l'étape juridictionnelle auprès du juge administratif.

Ainsi, le contentieux pénitentiaire se compose de deux phases, l'une préjuridictionnelle, lorsque la demande est adressée à l'Administration pénitentiaire, et l'autre juridictionnelle, lorsque la demande est adressée au juge administratif.

Ces deux phases sont distinctes mais pas dissociables dans la mesure où, la seconde peut constituer, normalement, le prolongement de la première. Les termes « contentieux public pénitentiaire », ou « contentieux carcéral » pourront être indistinctement employés ${ }^{16}$. A ce moment-là, le juge administratif est désigné compétent pour contrôler la légalité de l'activité pénitentiaire. Les litiges relatifs à la nature et aux limites des peines privatives de liberté relèvent de la compétence du juge judiciaire alors que le juge administratif doit connaître des litiges relatifs au fonctionnement administratif du service public pénitentiaire. Ainsi, la détermination de l'ordre juridictionnel n'a pas été suivie de la mise en œuvre immédiate du contrôle de légalité des actes pris par l'administration pénitentiaire.

L’Ordonnance n`344 du 17 septembre 1965 portant régime pénitentiaire congolais en vigueur jusqu'à ce jour, posant les bases législatives d'un Droit pénitentiaire dont la qualité, est-il vrai, doit être nuancée ${ }^{17}$.

14 A.COCATRE-ZILGEN, « La nature juridique des mesures d'ordre intérieur en droit administratif français», in RISA 1958, p. 487.

15 «La notion de contentieux recouvre le droit des litiges au sens large. On comprend par là non seulement les litiges devant un juge, mais aussi ceux sans juge ». G. KALFLECHE, "La notion de contentieux, une spécificité du droit public ", in, L'identité du droit public, X. Bioy, Presse de l'université Toulouse 1 Capitole, Actes de colloques $n^{\circ}$ 9, mars 2011, p. 159.

16 Le contentieux pénitentiaire peut également se distinguer du point de vue de son caractère disciplinaire ou extra-disciplinaire. Les contestations pré-juridictionnelles disciplinaires, proches $d u$ droit pénal, bénéficient d'un encadrement juridique " ancien »125 et, en théorie au moins, efficacement protecteur des droits des personnes détenues. Malheureusement, ce cadre juridique se heurte à de nombreux obstacles pratiques. En revanche, les contestations d'ordre non disciplinaire bénéficient d'un cadre juridique plus souple et moins précis, plus facilement respecté, dans sa mise en ouvre, par l'administration pénitentiaire. L'existence d'un cadre juridique [trop] strict ne l'incite pas à l'appliquer, alors qu'une certaine liberté d'action peut parfois l'inciter à prendre des précautions non prévues. En outre, le fait que ces phénomènes opposés s'expriment dans des domaines distincts, disciplinaire et non disciplinaire, conduit à accentuer l'appréciation du décalage entre le droit et sa pratique.

17 L'Ordonnance $n^{\circ} 344$ du 17 septembre 1965 est le texte principal en matière pénitentiaire en RDC. Elle organise, entre autres, l'administration des établissements pénitentiaires et établit le régime des détenus, notamment en matière d'hygiène, de soins médicaux et de nourriture ainsi de suite. 
Ainsi, il devient possible d'évoquer l'élaboration d'un statut juridique du détenu composé de droits et obligations, et susceptible d'emprunter plusieurs figures, notamment celle de la personne humaine, du citoyen, du justiciable et de l'usager contraint. La personne détenue devient titulaire de droits et destinataire de prestations fournies par le service public et, dispose de voies de Droit pour les faire respecter.

S'agissant des recours juridictionnels, les personnes détenues peuvent emprunter les voies classiques de contestation. D'abord, le recours pour excès et/ou abus de pouvoir pourrait permettre une amélioration des droits des détenus. Mais l'efficacité de ce recours serait désormais confrontée aux limites du contrôle restreint opéré par le juge. Ensuite, la définition de la faute pénitentiaire pourrait aussi élargir le champ de rayonnement du recours en responsabilité contre l'Administration pénitentiaire, même si le pragmatisme du Ministère public tend à en limiter la portée ${ }^{18}$.

L'Administration pénitentiaire ne fait pas systématiquement preuve de rigueur dans le maniement des procédures prévues pour chaque matière. Il peut arriver d'ailleurs qu'elle sanctionne l'indiscipline carcérale par le biais des sanctions pénitentiaires « occultes » ou « déguisées $»^{19}$.

\section{B. L'ACCES AU DROIT ET LES VOIES DE RECOURS PREALABLES AUX DETENUS.}

L'évolution du statut de détenu requérant n'est pas caractérisée par l'amélioration constante des droits afférents, dans la mesure où la protection effective des droits du demandeur ne suit pas toujours leur reconnaissance. L'accès au recours des détenus signifie, l'utilisation possible des moyens juridiques de droit public, de l'accès au droit en détention à l'utilisation des recours administratifs puis juridictionnels, en passant par les voies juridiques indirectes moins connues. L'accès signifie également que devra être déterminée l'effectivité de ces recours, et leur efficacité, en d'autres termes leur capacité à prospérer lorsqu'ils sont utilisés par les personnes détenues ${ }^{20}$.

Le droit de la détention regroupe l'ensemble des dispositions applicables au sein de l'établissement pénitentiaire et contenu dans divers instruments juridiques tant législatifs que règlementaires. La connaissance de ce droit par les personnes incarcérées est essentielle au bon fonctionnement de l'établissement. Elle permet au détenu de faire ses demandes dans la limite des prescriptions juridiques, tout en encadrant les pouvoirs dévolus à l'Admi-

18 M. NIEVE, "Droit des détenus : sens et non-sens de la prison, un état des lieux du droit pénitentiaire en Belgique », in, "Lette Actualités Droits-Libertés », CREDOF, 26 mars 2013.

19 M. HERZOG-EVANS, "Les sanctions disciplinaires occultes », in, La sanction du droit, Mélanges offert à P. Couvrat, p.u.f, 2001, p. 471 et s.

20 B. DE LAMY, « Le cadre constitutionnel du droit pénitentiaire », in RSC 2010, p. 217. 
nistration pénitentiaire. Or en République Démocratique du Congo, ces règles varient d'un établissement à l'autre ${ }^{21}$.

En effet, les conditions d'accès au droit en détention ne sont pas identiques dans les maisons d'arrêt, les centres de détention et au sein des établissements pénitentiaires. Les régimes de détention diffèrent en fonction de la population carcérale qui y est détenue. En outre, les pouvoirs discrétionnaires dévolus aux directions d'établissements pénitentiaires constituent des particularismes locaux qui ne permettent pas de faire une analyse complète de tous les dispositifs.

C'est pourquoi, nous nous attacherons à étudier le dispositif tel qu'il est prévu par le droit commun, applicable à minima à tous les établissements de la République Démocratique du Congo, et censé trouver un écho homogène sur l'ensemble du territoire congolais. La situation du détenu en République Démocratique du Congo présente une difficulté spécifique qui s'explique en raison de la dualité de notre système juridique et de nos voies de recours en matière de contentieux administratif.

Ainsi, la personne incarcérée se situe, en effet, dans une position juridique précise, celle de l'utilisateur, certes, contraint et forcé, d'un service public pénitentiaire. Les recours relatifs au fonctionnement de ce service public administratif relèvent classiquement de la compétence du juge administratif. Mais d'un autre côté, toutes les décisions qui concernent l'application et la durée de la peine privative de liberté ou la mesure de détention préventive, et qui sont prises par des magistrats de l'ordre judiciaire, relèvent de la compétence du juge judiciaire. Cette dualité de contentieux s'explique par le fait que le juge administratif est compétent pour connaitre des litiges relatifs à l'exécution du service public pénitentiaire par le personnel administratif et notamment par les directeurs des établissements pénitentiaires $^{22}$. Or, le juge congolais a été fort réticent à connaitre des requêtes émanant de personnes privées de leur liberté.

Ainsi, « destinées à assurer la discipline dans les établissements pénitentiaires ${ }^{23}$, les sanctions disciplinaires relevaient-elles, de cette catégorie, n'affectant pas le statut même des détenus et ne faisant donc pas grief? Sont ainsi toujours considérés comme des mesures d'ordre intérieur, les décisions de transfert et d'affectation ${ }^{24}$, le fichage d'un détenu comme détenu dangereux ${ }^{25}$, la décision de faire quitter à un détenu la cellule qu'il occupait... Il faut noter en conséquence que, le détenu usager du service public pénitentiaire n'apparait donc toujours pas en mesure de faire protéger l'ensemble de ses droits par des recours ou-

21 J-P CERE, "Le système pénitentiaire français 》, in les systèmes pénitentiaires dans le monde, sous la direction de J-P Céré, C-E. A. Japiassu, coll. Thèmes et commentaires, Dalloz, $2^{e}$ édition, Paris, 2011, p.169. Pour l'auteur, l'information permet d'améliorer le fonctionnement de l'établissement, en évitant des incidents puisés dans la méconnaissance de la réglementation ou en contribuant à atténuer le nombre de requêtes infondées.

22 FARGEAU D'epied Dame, cité par LARRALDE J.M., op-cit, p. 68.

23 Idem, p.69.

24 CE 8 Décembre 1967. KAYANAKIS; LARRALDE Jean-Manuel, op-cit, p. 68.

25 CE 8 Décembre 2000, FREROT; Ibidem, p. 68 
verts et accessibles. Le Droit pénitentiaire offre ainsi l'image d'une modernisation qui tend à faire du détenu un sujet de droit véritable. Toutefois, s'arrêter à des constatations n'aboutirait qu'à percevoir une partie de la réalité, car la situation du détenu ne doit pas être séparée des réalités carcérales qui aboutissent dans les faits, à la limitation de l'exercice des droits en détention.

L'accès au droit suppose que les personnes détenues puissent en prendre connaissance de façon effective, malgré la privation de liberté. Grâce à cette connaissance du droit qui lui est applicable, le détenu sera mieux armé pour faire face à toute violation de ses droits à la phase préjuridictionnelle.

La connaissance par la personne incarcérée de ses droits ainsi que des règles propres à l'établissement dans lequel il est incarcéré est un préalable nécessaire à toutes demandes faites de sa part, qu'elles soient administratives ou juridictionnelles. Le détenu requérant doit être en mesure d'emprunter les voies de droit qui lui sont offertes dans le respect du cadre légal et règlementaire qui s'impose à lui au cours de son incarcération. C'est pourquoi l'accès au droit de la personne détenue fait désormais l'objet d'un dispositif adéquat lors de son arrivée en détention, mais limité en cours de détention, puisque malgré les interventions du législateur, le détenu est sans cesse confronté à des limites résultant de sa privation de liberté.

S'il est juridiquement encadré, l'accès au droit en détention et l'accès au droit de la défense en milieu pénitentiaire s'améliorent. Toutefois, de nombreux obstacles demeurent, puisque ce dispositif se heurte toujours à la qualité de la norme pénitentiaire ou à son application «sui generis», sous-entendu adaptée à l'institution carcérale, nécessitant des précisions $^{26}$. Cependant, l'accès au droit n'est pas suffisant. Les personnes privées de liberté doivent en outre bénéficier de voies de droit utiles à la protection de leurs droits et bénéficier ainsi comme tout justiciable, de recours permettant une telle protection.

La personne privée de liberté dispose donc des droits de recours administratif, de procédures de référés non urgents et d'une voie de droit spécialement dédiée au contrôle général des lieux de privation de liberté. Mais ces voies de droit potentielles ne contribuent pas à part égales à l'amélioration des droits du détenu requérant. Elles sont en outre caractéristiques de la situation des personnes incarcérées dont la protection des droits procéduraux induit, insidieusement, l'application d'un régime adapté. La connaissance des droits en prison est une problématique récurrente dont l'amélioration constante se heurte inévitablement à la qualité de la norme. C'est à ce paradoxe que tentent de répondre les acteurs du monde carcéral. L'utilisation par les détenus de leurs droits tant matériels que procéduraux, passe nécessairement par la connaissance qu'ils peuvent avoir de leurs droits.

En effet, pour qu'un justiciable, libre ou privé de liberté, puisse demander la protection de ses droits, il doit d'abord les connaître. Or la situation privative de liberté de la personne

26 F. SAINT-PIERRE, et S. CORMIER, "L'avocat face à la nouvelle loi pénitentiaire », in RPDP, 2010, p. 107. 
détenue pose, de ce fait, la question d'une telle connaissance à travers la question de l'accès au droit en détention.

L'accès au droit en détention suppose également que les détenus aient accès aux droits de la défense en détention. La connaissance et l'utilisation de ces derniers sont indispensables pour faire face à l'Administration. Certains auteurs parlent « d'accès à l'information du détenu pour la préparation de sa défense ». Ce droit à l'information fait référence à la connaissance par le détenu des droits liés à sa situation de requérant au sein de l'établissement. Or ces droits doivent être respectés par l'administration pénitentiaire lorsqu'elle édicte certains actes ou met en œuvre certaines procédures internes ${ }^{27}$.

Ainsi, la connaissance par la personne incarcérée de ses droits ainsi que des règles propres à l'établissement dans lequel elle est incarcérée est un préalable nécessaire à toutes demandes faites de sa part, qu'elles soient administratives ou juridictionnelles. Le détenu requérant doit être en mesure d'emprunter les voies de droit qui lui sont offertes dans le respect du cadre légal et règlementaire qui s'impose à lui au cours de son incarcération. C'est pourquoi, l'accès au droit de la personne détenue fait désormais l'objet d'un dispositif adéquat lors de son arrivée en détention, mais limité en cours de détention, puisque malgré les interventions du législateur, le détenu est sans cesse confronté à des limites résultant de sa privation de liberté.

L'Administration pénitentiaire doit, au cours d'entretiens réalisés pendant la période d'accueil, informer oralement le détenu du cadre normatif qui s'impose à lui. Cela suppose d'abord de l'informer quant au régime de détention qui lui est applicable. Autrement dit, elle doit porter à sa connaissance les droits auxquels il peut prétendre et les obligations qu'il doit respecter. L'information orale sur les différents régimes de détention est essentielle puisque le choix de tel ou tel régime conditionne l'accès au droit des détenus au cours de leur détention ${ }^{28}$.

En effet, en fonction du régime strict ou ouvert qui leur est applicable, les détenus pourront ou non, sortir de leur cellule, circuler dans l'établissement ou accéder librement aux activités au sein de la prison. L'Administration doit également informer le détenu des modalités d'organisation et de fonctionnement de l'établissement.

Les règles de vie quotidiennes qui régissent la vie de l'établissement ainsi que le fonctionnement des différents services auxquels le détenu est susceptible de s'adresser lors de

27 M. GUYOMAR, "Limites des mesures d'ordre intérieur en matière pénitentiaire : déclassement d'emploi et changement d'affectation des détenus ", in Concl. Sur, CE. Ass, 14 décembre 2007, M. Planchenault et M. Boussouar, RFDA, 2008, p.94.

28 Dès leur accueil dans l'établissement pénitentiaire et à l'issue d'une période d'observation pluridisciplinaire, les personnes détenues font l'objet d'un bilan de personnalité. Un parcours d'exécution de la peine est élaboré par le chef d'établissement et directeur du service pénitentiaire d'insertion et de probation pour les condamnés, en concertation avec ses services, dès que leur condamnation est devenue définitive. La répartition des condamnés dans les prisons s'effectue compte tenu de leur catégorie pénale, de leur âge, de leur état de santé et de leur personnalité. Leur régime de détention est déterminé en prenant en compte leur personnalité, leur santé, leur dangerosité et leurs efforts en matière de réinsertion sociale. 
l'exécution de la peine doivent lui être communiqués. Dès lors, il est permis d'affirmer qu'en informant oralement l'arrivant des spécificités de l'établissement, les entretiens contribuent indéniablement à améliorer l'accès au droit des détenus. Les entretiens d'accueil demeurent cependant une étape importante qui contribue incontestablement à améliorer l'accès au droit de la personne nouvellement détenue. Pour preuve, ces entretiens peuvent s'étaler entre une et trois semaines, et sont menés par des agents spécialement formés ${ }^{29}$.

La nécessité pour les détenus de connaître le contenu du règlement intérieur et d'y avoir effectivement accès est, selon nous, plus importante en cours de détention que dès son arrivée au sein de l'établissement. En effet, l'information délivrée lors de la phase d'accueil permet une première prise de contact générale et complète de la norme carcérale. Mais ce dispositif d'accueil efficace, demeure une utopique dans les établissements pénitentiaires congolais alors que pendant l'incarcération, l'information transmise doit nécessairement être actualisée et continue. De plus, la discipline pénitentiaire fait l'objet d'une évolution jurisprudentielle constante, de plus en plus protectrices des droits des détenus. Toutefois, l'amélioration du cadre juridique ne peut masquer l'insuffisance des droits protégés, d'autant que ceux garantis sont difficilement mis en œuvre.

En effet, la nature juridique de sanctions disciplinaires en détention " mixte " et son fonctionnement «opaque » peut rendre difficile et insuffisante la garantie des droits de la personne détenue. Ainsi, la discipline carcérale, matériellement pénale, mais procéduralement administrative, peut être définie comme « l'ensemble des règles de conduite imposées aux membre d'une collectivité pour assurer le bon fonctionnement de l'organisation sociale ». Autrement dit, c'est un mécanisme de gestion et de contrôle de la population carcérale. Dès lors, sa nature répressive devrait justifier, la reconnaissance et le respect de droits et garanties incontournables : une procédure disciplinaire équitable, contradictoire et impartiale, respectueuse des droits de la défense ${ }^{30}$.

La personne détenue bénéficie-t-elle d'une procédure équitable, contradictoire et impartiale? Dispose-t-elle de moyens juridiques efficaces pour faire face à l'omnipotence de l'administration pénitentiaire et son pouvoir discrétionnaire? A ce titre, se pose également la question de la composition de la commission de discipline, ainsi que sa nature? Les voies de contestations sont-elles respectueuses des droits de la défense? Permettent-elles de faire utilement prospérer les demandes? Ainsi la question de l'étendue de la reconnaissance, de leur respect et de la mise en œuvre des droits de la personne détenue, se pose tout au long du déroulement de la procédure disciplinaire.

Par ailleurs, le respect de la règle de droit suppose qu'elle soit connue des destinataires auxquels elle s'applique, y compris pour les personnes privées de liberté. C'est la conclusion tirée de l'arrêt de la Cour administrative d'appel de Paris, en date du 29 juin

29 C. VIGOUROUX, «La valeur de la justice en détention », in AJDA, 2009, p.403.

$30 J-M$ PASTOR, "Dignité du régime de détention et office du juge du référé provision », in AJDA, 2013, p. 246. 
2001, au sujet de la connaissance par les détenus des règlements applicables au sein des établissements pénitentiaires. En l'espèce, les juges ont considéré, à propos d'une circulaire règlementaire relative à la fouille des détenus, que n'ayant " fait l'objet d'une quelconque publication au Journal officiel de la République française ou d'une mesure de publicité adéquate au sein de la maison d'arrêt de Fresnes; qu'ainsi faute de publicité suffisante, cette circulaire n'était pas opposable à $M . X \ldots »^{31}$.

Nous estimons que le juge doit imposer à l'Administration pénitentiaire de mettre en œuvre les formalités nécessaires à la publicité des actes réglementaires pénitentiaires, sous peine de les rendre inapplicables. Il peut s'agir d'une parution au journal officiel ou d'une mesure de publicité appropriée au sein de l'établissement de détention et dont les modalités sont laissées à la discrétion de l'Administration. Dès lors, il est permis d'affirmer que la privation de liberté ne peut suffire à justifier des dérogations au principe de l'accès au droit, qui impose clairement la publicité des actes règlementaires pénitentiaires au profit des personnes incarcérées.

Dans nos analyses en sus, il est démontré qu'il existe un véritable décalage entre les textes et la pratique. La pratique du contentieux disciplinaire ne s'est jamais exercée dans nos cours et tribunaux car la mise en œuvre du cadre juridique ne permet pas de garantir effectivement les droits qu'il reconnaît.

De nombreuses incertitudes subsistent quant à la reconnaissance, la protection et l'exercice des droits du détenu justiciable face aux sanctions disciplinaires. Lorsqu'un détenu commet un acte contraire aux dispositions internes à l'établissement pénitentiaire, il fait souvent l'objet d'une procédure disciplinaire. Cette procédure commence par l'accusation par le surveillant témoin des agissements considérés comme fautifs. Cela suppose que la personne détenue ne bénéficie pas de garanties lors de son accusation et le principe de la présomption d'innocence est mis à mal.

A notre avis, l'exercice du recours préalable a, s'il est mis en œuvre, peu de chance d'aboutir dans la mesure où l'autorité hiérarchique (directeur de prison) désavoue rarement son subordonné. Nous proposons à la place le recours juridictionnel, cela reviendrait à affaiblir l'autorité du chef d'établissement auprès du personnel pénitentiaire et des détenus. Néanmoins, cela ne doit pas décourager les détenus qui, bien qu'ayant peu de chance de voir leur contestation aboutir, bénéficient d'autres moyens juridiques pour faire prospérer leur demande.

\section{L'EFFICACITE MESUREE DES RECOURS JURIDICTIONNELS COMME MECANISME DE PROTECTION DES DROITS DES DETENUS.}

Il est impérieux que le juge congolais entre en prison pour veiller de manière ferme mais équilibrée au bon fonctionnement de ce service public pénitentiaire. Il s'attachera au respect

31 CAA Paris, plénière, 29 juin 2001, Frérot, req. $n^{\circ} 97 P A 03554$ et $n^{\circ}$ 97PA03556, inédit au Rec. Lebon; J.-P. CERE, M. HERZOG-EVANS, E. PECHILLON, «Droit de l'exécution des peines : panorama 2005 », Rec. Dalloz 2005, p. 1085. 
du droit et des obligations applicables en détention. Il affirmera que les mots ne doivent pas être détournés et qu'un droit est un droit quel que soit le côté du mur où l'on se trouve. La personne détenue est un justiciable.

Si cette affirmation est aujourd'hui permise, tel n'a pas toujours été le cas en Droit congolais. Les personnes incarcérées ont longtemps été soumises à l'ordre intérieur carcéral, sans bénéfice de la protection légale générale que peut assurer le juge congolais, réticent à pénétrer la société carcérale.

L'entrée du Droit en prison peut progressivement contraindre à contrôler la légalité du fonctionnement de l'activité du service public pénitentiaire. Le développement des recours au profit des détenus pourra permettre l'affirmation de son contrôle juridictionnel. Ainsi, le juge devra ainsi devenir le « bras armé » du détenu, susceptible d'élever le niveau de protection de ses droits. Il analysera le droit existant, le complète, et en contrôle son respect en détention.

Pour demander au juge de contrôler la prison et le respect des droits, le détenu dispose, comme tout justiciable, des différents recours juridictionnels devant le juge. En détention, il peut être distingué, le recours pour excès de pouvoir contre les sanctions disciplinaires, des recours en responsabilité, des recours en référés urgents utilisables dans les domaines non disciplinaires ${ }^{32}$.

Cette distinction n'est ni impérative, elle découle de notre choix de plan et de sa justification, ni parfaite dans la mesure où le contentieux objectif et les référés peuvent également rayonner en contentieux disciplinaire, dans ce cas pour obtenir une décision autre qu'une déclaration d'illégalité. En outre, cette distinction s'atténue lorsque l'on examine d'éventuelles pratiques illégales de l'Administration pénitentiaire visant à réprimer indirectement l'indiscipline carcérale par le biais des sanctions pénitentiaires occultes ou déguisées ${ }^{33}$. Celle-ci utilise le droit pénitentiaire commun, juridiquement plus souple, pour contourner le régime juridique prévu pour la répression disciplinaire au sein du service public, lequel obéit à un régime juridique beaucoup plus contraignant.

L'unique étude du recours pour excès de pouvoir en matière disciplinaire permet d'établir un constat d'insuffisante efficacité de cette voie de Droit, malgré son accessibilité certaine.

En effet, l'encadrement réglementaire et jurisprudentiel ancien du Droit disciplinaire, permet de dire que ce recours est aujourd'hui obsolète. En revanche, étudier ce recours en matière non disciplinaire aurait tendance à ne permettre d'établir qu'une liste énumérative, peu utile à notre démonstration.

32 G. FAUGERE, L'accès des personnes détenues aux recours. Etude de Droit administratif, Thèse de doctorat en Droit public, Université de Toulouse 1 Capitole, 30 Janvier 2015, inédit, p.277.

33 Le recours pour excès de pouvoir peut être évoqué à l'occasion de l'application des droits de la défense aux mesures d'isolement, de transfert, de mise en æuvre des régimes différenciés et de fouilles à nu systématiques. Mais dans cette étude il n'est nullement besoin d'approfondir cette question. 
Ces trois recours ont pour objectif de contrôler le respect du droit en détention, et ainsi réduire le décalage constaté à l'occasion de la phase pré- juridictionnelle. Celle-ci est marquée par la différence entre l'énoncé théorique des droits issus des textes, et leur application pratique quotidienne. La phase de contestation en détention, n'offre pas aux détenus une protection juridique suffisante et systématiquement mise en œuvre ${ }^{34}$.

Les différents contrôles à opérer au titre de chaque recours, et leurs effets, conduiront à qualifier le juge congolais de faiseur d'équilibre dans la mesure où, il arme autant l'Administration, qu'il protège les détenus. Il n'est ni « droit de l'hommiste », ni en faveur d'un contentieux verrouillé par le tout sécuritaire. Il adopte un raisonnement pragmatique, et apprécie avec réalisme la nécessité de protéger les droits des détenus, face à celle de garantir la sécurité de l'établissement et des personnes.

Dans ces conditions, les perspectives d'évolutions dépendront des détenus, de leur conseil et de la stratégie contentieuse adoptée face à l'Administration pénitentiaire, le juge étant neutre par nature. Il s'agit donc de déterminer si la personne détenue dispose réellement de garanties juridictionnelles effectives et efficaces, susceptibles de rééquilibrer le décalage constaté lors de la phase préjuridictionnelle et juridictionnelle. Le rééquilibrage espéré s'avère mesuré puisque l'accès au recours pour excès de pouvoir disciplinaire est limité par son manque d'efficacité, alors que les conditions d'exercice du recours seront trop strictement appréciées pour exprimer toutes leurs potentialités.

La théorie des mesures d'ordre intérieur a longtemps fait obstacle à l'examen juridictionnel des décisions prises en commission de discipline. De ce fait, c'est le régime juridique des sanctions disciplinaires carcérales que nous proposons. Partant, cela permettra progressivement d'élargir la recevabilité du recours pour excès de pouvoir contre l'ensemble des sanctions disciplinaires prononcées en commission de discipline en détention.

Il ressort de ce qui précède que les sanctions disciplinaires infligées aux personnes détenues relèvent, de façon certaine, de la catégorie des sanctions institutionnelles coercitives caractérisées par un lien de subordination. En effet, c'est sur ce lien que repose le pouvoir de sanction au sein des établissements pénitentiaires. La dépendance et la subordination des captifs est totale vis-à-vis de l'Administration ${ }^{35}$.

Le recours ouvert contre les sanctions carcérales est un recours pour excès de pouvoir. Cette voie de Droit est la voie classique de contestation de légalité des actes administratifs. Matérialisant « le procès fait à un acte », il possède de nombreux avantages, tant pour celui qui l'introduit, le détenu, que pour l'Administration pénitentiaire à l'origine de la décision sujette à contestation. Ainsi, il pourrait s'agir de la voie de contestation idoine pour les détenus indisciplinés ${ }^{36}$.

34 M. GUYOMAR, «Le juge administratif, juge du service public pénitentiaire », in, Les droits de la personne détenue. p. 155.

35 J.-M. DELARUE, "Chronique générale de jurisprudence administrative française ", AJDA 1984, p. 72.

36 F.MELLERAY, «Une nouvelle réduction du champ des mesures d'ordre intérieur en milieu carcéral», DA 2008, $n^{\circ} 2$, com $n^{\circ} 24$. 
Le basculement vers le contentieux des litiges relatifs aux sanctions disciplinaires pénitentiaires pourrait également être envisagé comme la proposition adéquate au rééquilibrage des droits de la personne détenue face à ceux de l'Administration pénitentiaire. Le juge congolais serait ainsi un juge mieux « partagé » entre la protection des droits du détenu indiscipliné et la prise en compte des exigences sécuritaires nécessaires au fonctionnement du service public pénitentiaire ${ }^{37}$.

Ainsi, le recours pour excès de pouvoir présente de nombreux avantages pour le justiciable qui l'exerce. La personne détenue qui conteste une sanction disciplinaire bénéficie de ses effets positifs puisqu'il contribue à faciliter l'accès au juge et à la protéger contre les sanctions manifestement illégales ${ }^{38}$.

Pour notre part, nous estimons que le recours pour excès de pouvoir possède une plus grande efficacité. Il est vrai que cette accessibilité se justifie par le fait que le recours en annulation est un recours objectif, dirigé contre un acte administratif unilatéral. Ce «procès fait à un acte », pour reprendre la formule classique mais appropriée, consiste en l'examen de la conformité de la décision avec la hiérarchie des normes. Il s'agit donc d'une question de droit objectif laquelle ne nécessite pas la démonstration d'un intérêt subjectif ou un préjudice particulier pour contester la sanction.

Le recours pour excès de pouvoir peut progressivement offert la possibilité aux personnes privées de liberté de contester toutes les sanctions disciplinaires pénitentiaires qui peuvent leur être infligées. Il en est de même des sanctions infligées par les autorités administratives dans le cadre d'un service public. Dès lors, le contentieux disciplinaire carcéral relèvera, sans aucun doute, du contentieux de l'excès de pouvoir ${ }^{39}$.

Ce recours possède une fonction répressive, et préventive dans la mesure où il permet d'orienter l'action pénitentiaire. Pour bénéficier de son efficacité, il appartient aux détenus d'apporter la preuve précise des conditions de mise en jeu de la responsabilité, ce qui est modérément facilité par la transformation de la faute pénitentiaire ${ }^{40}$.

La personne détenue devra, comme cela est traditionnellement admis, apporter la preuve de la responsabilité de l'État. Pour cela, elle doit démontrer que l'Administration a commis une faute lui ayant directement causé un préjudice indemnisable. Le régime juridique de la responsabilité administrative est donc soumis à la preuve de trois éléments : la démonstration d'une faute, d'un préjudice et d'un lien de causalité entre la faute et le préju-

37 H. BELRHALI-BERNARD, "Le juge administratif et le contrôle de l'administration pénitentiaire », in, L'administration pénitentiaire face aux principes de la nouvelle gestion publique, préc. p. 183.

38 S.-J. LIEBER, et D. BOTTEGHI, "L'étoile du recours pour excès de pouvoir pâlirait-elle encore? », AJDA 2009, p. 583.

39 E. LAFERRIERE, Traité de la juridiction administrative et des recours contentieux, Berger-Levrault, ler éd., Tome I, 1887 1888. L'auteur distingue les différentes branches du contentieux en fonction des pouvoir reconnus au juge administratif. Il divise le contentieux en quatre branches: Le contentieux de pleine juridiction, le contentieux de l'annulation, le contentieux de l'interprétation, et le contentieux de la répression.

40 D. BOUJU, «Le détenu face aux mesures d'ordre intérieur », in RDP 2005, $n^{\circ} 3$, p. 597. 
dice. Ce dernier doit être personnel, direct et certain, et être la conséquence directe d'une erreur de l'Administration dans la gestion du service public dont elle a la charge ${ }^{41}$.

Par ailleurs, la faute pénitentiaire a, dans un premier temps, connu une évolution lente mais constante ayant conduit à sa nouvelle définition ainsi qu'à l'évolution de son contenu. En effet, après avoir fait primer les contraintes sécuritaires sur les droits des personnes détenues, le juge peut progressivement considérer qu'elles bénéficiaient de droits, dont le nonrespect ne pouvait rester sans conséquence. La prise en compte des droits peut être rendue possible par l'encadrement juridique de l'activité pénitentiaire ${ }^{42}$, qui peut devenir un véritable service public.

Dans le même ordre d'idées, on peut prévoir également de nombreux droits au profit des personnes détenues. Ces dispositions peuvent contribuer à préciser la mission de service public pénitentiaire et par conséquent les droits des détenus. En effet, ce n'est qu'en pénétrant d'avantage l'institution pénitentiaire que les droits textuellement garantis pourront faire l'objet d'une amélioration effective dans la mesure où le contrôle juridictionnel est la garantie d'une reconnaissance accrue et d'une protection réelle des droits ${ }^{43}$.

Le juge congolais peut ainsi développer un contrôle plus large et plus précis des éléments qui permettent de qualifier un comportement de fautif au regard des obligations mises à la charge de l'Administration pénitentiaire.

L'objectif n'est pas de faire l'étude de la totalité du régime de responsabilité de l'Administration pénitentiaire. Il serait audacieux, et même présomptueux d'envisager une telle analyse au cours d'un article scientifique au regard de la complexité et de l'hétérogénéité de ce contentieux.

En outre, une telle étude n'aurait qu'une fonction énumérative, et ne permettrait pas de démonter en quoi la responsabilisation de l'Administration pénitentiaire a permis d'améliorer l'accès au juge du contentieux et donc d'améliorer des droits du détenu grâce à un rééquilibrage jurisprudentiel mesuré.

Il s'agit donc d'analyser les dommages causés aux personnes détenues par l'activité pénitentiaire, excluant de ce fait de notre étude une partie du contentieux de la responsabilité. De même, il ne s'agit pas ici de faire l'étude des responsabilités personnelles des agents pénitentiaires ou des intervenants au sein de la prison, mais bien uniquement celle de l'Administration pénitentiaire agissant au nom de l'État dans l'une de ses fonctions régaliennes les plus importantes. En d'autres termes, ce sont les fautes de service, relevant de l'institution, qui sont analysées et non les fautes personnelles. Enfin, il ne s'agit pas non plus de

41 A. Bretonneau, J. Lessi, "De l'opportunité et de la légalité en matière disciplinaire », in AJDA 2014, p. 1478.

42 V. J. Martinez-Mehlinger, "Vers l'atomisation du recours pour excès de pouvoir dans le contentieux des sanctions administratives », RFDA 2012, p. 258.

43 I. de Silva, "La rénovation du régime de responsabilité de l'État du fait des services pénitentiaires ",

in AJDA 2009, p. 416. 
faire l'étude de la responsabilité pénitentiaire sous l'angle du droit européen dont il est acquis que les dispositions assurent aux personnes détenues de solides garanties.

Ainsi finalement, si le juge peut rééquilibrer la balance au profit des détenus en généralisant la faute simple pénitentiaire à « tous les cas d'engagement de responsabilité de la puissance publique en matière pénitentiaire », il pourra également rééquilibrer au profit de l'Administration pénitentiaire en renforçant et en élargissant son contrôle des conditions de mise en œuvre de responsabilité. Il est ainsi permis d'affirmer que le cadre juridique de responsabilité demeure d'une part équilibré, et d'autre part, à fortes potentialités ${ }^{44}$.

En faisant de la faute simple le fait générateur de Droit commun de la responsabilité, le juge pourra faciliter l'engagement de la responsabilité de l'État du fait de son activité pénitentiaire. De larges potentialités s'offriront aux détenus dans la mesure où ils pourront prétendre à être indemnisés de l'ensemble des préjudices qu'ils subissent. Toutefois, cette évolution ne peut avoir pour conséquence l'engagement « illimité » d'une telle responsabilité ${ }^{45}$. De ce fait, le juge congolais pourra ainsi faire évoluer son contrôle, en d'autres termes, adapter son analyse au nouveau contexte, afin d'assurer le maintien de l'équilibre entre le respect des droit des détenus et les contraintes inhérentes au fonctionnement du service public pénitentiaire.

Le recours de contentieux et les procédures de référés urgents sont au nombre des voies de contestation accessibles aux personnes détenues. L'étude de la jurisprudence a permis de démontrer que leur existence et leur efficacité n'est pas illusoire, même s'il est vrai que leur exercice est systématiquement adapté à la détention ${ }^{46}$.

Le juge étant pragmatique, devra prendre l'habitude d'examiner précisément tous les éléments rapportés et susceptibles de l'aider dans son contrôle. Il semble d'ailleurs ne pas limiter son examen à tels ou tels éléments, facilitant ainsi l'obligation de preuve des personnes détenues. La difficulté pour eux, réside d'avantage dans le fait qu'il leur est délicat d'obtenir ces éléments de preuve, en détention, dans la mesure où ils sont tributaires de l'Administration pénitentiaire qui a tendance à faire primer les contraintes sécuritaires, fausses ou avérées, sur la protection de leurs intérêts ${ }^{47}$.

Pour clore ce chapitre, il faut noter que le recours pour excès de pouvoir tel qu'il est admis contre les sanctions disciplinaires pénitentiaires ne permet pas de garantir efficacement la protection des droits des détenus et le contrôle de l'activité disciplinaire en détention. L'évolution du recours contre cette catégorie particulière d'actes administratifs unilatéraux s'articule donc autour de deux idées.

44 M. MOLINER-DUBOST, "A propos d'une autre " jurisprudence immobile ». Le contentieux des sanctions disciplinaires infligées aux détenus », in AJDA 2013, p. 1383.

45 N. FERRAN, "La personne détenue encore à la recherche de son juge », in, Défendre en justice la cause des personnes détenues, CNCDH, préc., p. 140.

46 P. PONCELA, "Discipline pénitentiaire, un droit en mouvement », RSC 2012, p. 216.

47 J.-M. SAUVE, "Le contrôle de l'administration pénitentiaire par le juge administratif », 24 février 2009, in, J.-B. Auby, Le juge administratif, juge pénitentiaire, DA 2014, $n^{\circ} 3$, repère 3. 
La première idée repose sur le fait que les sanctions infligées aux personnes détenues doivent désormais faire l'objet d'un contrôle juridictionnel approfondi, dans la mesure où l'examen du juge ne correspond plus aux évolutions constatées en droit pénitentiaire et en droit de la sanction disciplinaire. La seconde idée repose sur le fait qu'en rejetant la solution d'un contrôle de plein contentieux, il devient indispensable de trouver une solution intermédiaire entre, les perspectives limités du recours pour excès de pouvoir et le passage en plein contentieux.

C'est pourquoi, selon nous, un recours intermédiaire peut être approprié. Il consiste à faire du contrôle normal de proportionnalité, le nouveau contrôle juridictionnel des sanctions disciplinaires, dont la mise en œuvre s'accompagnerait d'un renforcement des pouvoirs du juge de l'excès de pouvoir.

\section{CONCLUSION}

L'étude de la juridictionnalisation du Droit pénitentiaire suppose la reconnaissance, d'une part de moyens matériels, et d'autre part, des droits procéduraux. Ces derniers s'apparentent comme la première étape nécessaire à la protection de

l'ensemble des droits fondamentaux de la personne détenue puisqu'ils sont indispensables pour mettre en œuvre la protection des droits matériels. Avec l'encadrement juridique de la détention, le statut de justiciable peut, peu à peu, être octroyé aux personnes détenues.

La reconnaissance de droits en détention et l'élaboration de moyens juridiques pour leur protection en attestent. Pourtant, malgré cela, les personnes détenues ne bénéficient toujours pas, de façon claire et précise, de l'ensemble des outils nécessaires à l'effectivité d'un tel statut.

L'accès au droit, l'accès au juge et le droit à l'assistance d'un conseil en détention bénéficient d'un régime juridique peu favorable. Par ailleurs, ce régime, dont les manifestations sont confrontées aux particularismes carcéraux, évoluent au rythme des interventions législatives mais surtout juridictionnelles.

Le contentieux public pénitentiaire se compose de deux phases. Le détenu bénéficie, devant le juge, d'une protection juridique de ses droits de plus en plus avancée. En revanche, son statut de personne privée de liberté conduit d'autre part, lors de la phase préalable au prétoire, au fait que ses droits afférents à sa qualité de demandeur soient adaptés à l'univers carcéral.

L'étude de la juridictionnalisation de l'exécution de la peine privative de liberté fondée ici sur l'accès des personnes détenues aux voies de recours, permet d'affirmer qu'il est possible d'une amélioration des moyens juridiques de contestation. L'amélioration théorique de ces moyens de Droit se heurte à une mise en œuvre pratique peu favorable aux reclus.

Déjà, la phase préjuridictionnelle offre, dès le début du parcours contentieux, des garanties limitées par l'enfermement. L'accès matériel et intellectuel au Droit pénitentiaire, qualifié de complexe, est insusceptible de permettre une connaissance suffisante du contenu de 
ce Droit. De plus, le Droit positif congolais n'encadre pas la phase disciplinaire en détention qui se heurte à deux écueils.

Le premier réside dans l'insuffisant respect des garanties juridiques. Le statut et le fonctionnement de la commission de discipline pénitentiaire permettent à cette dernière de ne pas mettre en œuvre les garanties indispensables de cette procédure. Il s'agira d'un procès pénitentiaire dont sont exclues des garanties juridiques, notamment relatives aux droits de la défense, pourtant nécessaires au vu de sa coloration pénale. Et le second réside dans l'ineffectivité pratique des droits reconnus.

L'opacité de la procédure disciplinaire au sein des prisons congolaises et la présence quasi inexistante des avocats en détention permet au directeur des prisons de diriger discrétionnairement toutes les actions en détention, et pas toujours conformément à l'état de Droit. Les moyens reconnus aux personnes détenues ne permettent pas de rétablir ce décalage entre le Droit et la pratique. Malheureusement encore, d'autres mesures non disciplinaires, telles que les mesures d'isolement, les mesures de transferts ou de fouilles sont prises en violation de droits de la défense. En outre, les possibilités de faire appel au Contrôleur des lieux de privation de liberté et au Défenseur des droits de l'homme sont occultées. C'est pourquoi, l'amélioration des recours juridictionnels est, à notre avis, la seule issue de secours à la disposition des personnes détenues pour faire respecter leurs droits en détention.

Le juge congolais, réaliste et pragmatique peut pleinement se saisir du contentieux carcéral. Il constitue une véritable garantie du Droit pénitentiaire et fera naître un nouvel espoir au profit des personnes détenues. Ainsi, l'efficacité des recours juridictionnels doit se fonder sur le respect de la Constitution et les textes des lois qui régissent les établissements pénitentiaires en RD Congo.

La recevabilité du recours pour excès de pouvoir contre les sanctions disciplinaires et autres abus de droit en détention se heurte à l'examen limité de la légalité de ces sanctions par le juge congolais, justifiant une redéfinition du régime juridique de ce recours. Le juge doit franchir le pas d'un contrôle renouvelé. Il doit pouvoir contrôler plus intensément la légalité des actes posés en détention et les juger en fonction des circonstances de droit et de fait existants le jour où il se prononce.

Il me semble également nécessaire d'assouplir les conditions d'exercice des différents recours utilisables par les personnes détenues. Les caractéristiques spécifiques de la détention justifient que la faute pénitentiaire et l'urgence pénitentiaire soient adaptées au contexte dans lequel elles sont analysées par le juge. Ce dernier doit devenir un faiseur d'équilibre qui arme autant l'Administration qu'il protège les détenus.

Pour atteindre un tel équilibre, cette protection doit être appréciée sous le prisme de la privation de liberté qui engendre nécessairement vulnérabilité, infériorité, absence d'autonomie, et limitation par nature des droits des personnes détenues. S'il peut être admis une restriction des droits matériels eu égard aux contraintes inhérentes à la détention, il ne peut $\mathrm{y}$ avoir de restriction aux droits procéduraux dans la mesure où, ils constituent le moyen indispensable à la protection des droits matériels des personnes détenues. 
L'amélioration des droits des personnes détenues devra également s'accompagner d'une amélioration des conditions matérielles de détention. La surpopulation carcérale et l'état vétuste des établissements pénitentiaires apparaissent comme des obstacles récurrents à une amélioration effective des droits des détenus. Le contentieux pénitentiaire sera un contentieux jeune, varié et spécialement complexe.

La confrontation entre le droit à l'exercice des recours, dont l'objet est de garantir le respect des droits, et la privation de liberté, laquelle limite de fait, l'exercice des droits, suggère l'existence de possibilités sous estimées et de potentialités encore à découvrir. Ces perspectives contentieuses sont renforcées par le constat, il est vrai peu flatteur, du nombre de personnes écrouées et détenues dans les prisons congolaises ${ }^{48}$.

La présence des avocats au sein des établissements pénitentiaires en apparaît alors comme essentielle pour que l'arme du Droit, associée à une stratégie contentieuse, permette l'affirmation et l'effectivité du respect des droits des personnes détenues. Si la prison est une nécessité pour la République Démocratique du Congo, au sein de laquelle doit régner un ordre juste, les contraintes carcérales doivent être limitées aux seuls effets directs de la privation de liberté et ne peuvent en aucun cas entraver le droit de contestation juridique des personnes détenues.

\section{BIBLIOGRAPHIE SELECTIVE}

\section{TEXTES DE LOIS ET DOCUMENTS OFFICIELS}

Constitution de la République Démocratique du Congo, in journal officiel, $52^{e}$ année, numéro spécial, Kinshasa, le 18 Février 2006 telle que modifiée en 2011.

Déclaration Universelle des droits de l'homme, New York, 10 décembre 1948

Ordonnance $n^{\circ} 344$ du 17 septembre 1965 portant régime pénitentiaire congolais.

\section{OUVRAGES CONSULTES}

AUBY, J-M., Le contentieux du service public pénitentiaire », in RDP, $n^{\circ} 103,1987$, p.547.

BELRHADI-BERNARD, H., "Quand l'obligation in solidum des coauteurs progresse en droit administration », in AJDA, 2011, p.116.

BELRHADI-BERNARD, H., "Le juge administratif et le contrôle de l'administration pénitentiaire », in, FRMENT J-C, KALUSZYNSKY M., L'administration pénitentiaire face aux principes de la nouvelle gestion publique, PUG, 2011, pp.175 - 186 .

48 L'enquête socio-juridique menée à la prison centrale de Kasapa, de décembre 2016 au janvier 2018 sur le respect du droit des détenus et son effectivité en milieu carcéral congolais s'est étalée sur une période de douze mois. Au cours de cette enquête, les effectifs de départ étaient 2.135 détenus dont 53 femmes en décembre 2016, sont passés au 05 Janvier 2018 à 2.389 détenus parmi lesquels 1.265 prévenus devant les juridictions de jugement, 140 hébergés, 78 ayant interjeté appel, 67 vagabonds, 35 mineurs (mesures de sûreté), 29 détenus politiques et 775 condamnés à la peine de servitude pénale principale. 
Juridictionnalisation du droit penitentiaire congolais: défis et perspectives.

BOUJU, D., "Le détenu face aux mesures d'ordre intérieur », in RDP, $n^{\circ}$ 3, 2005, p. 2353.

BRETONNEAU, A. et LESSI, L., "De l'opportunité et de la légalité en matière disciplinaire », in AJ$D A, 2014$, p.1484.

CARTIER M-E, «La Judiciarisation de l'exécution des peines », Rev. Sci. Crim, 2001.

CERE, J-P., «Le système pénitentiaire français », in les systèmes pénitentiaires dans le monde, sous la direction de J-P CERE, C-E. A. Japiassu, coll. Thèmes et commentaires, Dalloz, 2e édition, Paris, 2011.

CERE, J-P., HERZOG-EVANS, M. et PECHILLON, E., Droit de l'exécution des peines, Dalloz, Paris, 2005.

COCATRE-ZILGEN, A., " La nature juridique des mesures d'ordre intérieur en droit administratif français», in RISA, 1958, p. 487.

DE LAMY, B., "Le cadre constitutionnel du droit pénitentiaire », in RSC 2010, p.217.

DE SILVA, I., "La rénovation du régime de responsabilité de l'État du fait des services pénitentiaires », in AJDA, 2009, p.416.

DELARUE, J-M., " Droit et procédure en détention », Défendre en justice la cause des personnes détenues, in CNCDH, éd. La documentation française, Paris, 2014, p.80.

FAUCHER, P.; La juridictionnalisation de l'application des peines, une révolution tranquille, RPDP, 2001.

FAUGERE, G., L'accès des personnes détenues aux recours. Etude de Droit administratif, Thèse de doctorat en Droit public, Université de Toulouse 1 Capitole, 2015, inédit.

FERRAN, N., "Transfert entre établissement de même nature : petite avancée e », grand recul pour les droits des détenus », in, Dedans-Dehors, $n^{\circ} 82$, décembre 2013, p.46.

GUYOMAR, M., "Le juge administratif, juge du service public pénitentiaire », in Terres de droit, mélanges en l'honneur de YVES JEGOUZO, Dalloz, Paris, 2009, p.471.

GUYOMAR, M., "Limites des mesures d'ordre intérieur en matière pénitentiaire : déclassement d'emploi et changement d'affectation des détenus », Concl. Sur, CE. Ass, 14 décembre 2007, M. Planchenault et M. Boussouar, in RFDA, 2008, p.87.

HERZOG-EVANS, M., "Les sanctions disciplinaires occultes », in, La sanction du droit, Mélanges offert à P. COUVRAT, P.U.F, 2001, p.471.

HERZOG-EVANS, M., "Reconnaissance et limites des recours des détenus 》, $2^{e} e ́ d$. Dalloz, Paris, 2008.

KALFLECHE, G., "La notion de contentieux, une spécificité du droit public », in, L'identité du droit public, Bioy, X., Presse de l'université Toulouse 1 Capitole, Actes de colloques $n^{\circ}$ 9, mars 2011, p.159.

KALUSZYNSKY, M., L'administration pénitentiaire face aux principes de la nouvelle gestion publique, PUG, 2011.

Ordonnance $n^{\circ} 344$ du 17 septembre 1965.

LAFERRIERE, Traité de la juridiction administrative et des recours contentieux, Berger-Levrault, ler éd., Tome I, 18871888.

LARRALDE J.M., "Les droits des personnes incarcérées : entre punition et réhabilitation », in CRDF, 2003, $n^{\circ} 2, p .63$. 
LECOINTE VIRGINIE, La juridictionnalisation de l'exécution des peines, Mémoire d'études approfondies, Ecole doctorale $n^{\circ} 74$, Faculté des sciences juridiques politiques et sociales, Université de Lille II, Droit et Santé, 2001-2002.

LIEBER, S-J., et BOTTEGHI, D., "L'étoile du recours pour excès de pouvoir pâlirait-elle encore? ", in AJDA 2009, p.583.

LORCY, M., "L'évolution des conceptions de la peine privative de liberté », in CRDF, 2004, $n^{\circ}$ 3, p.11.

MARTUNEZ-MELHINGER, J., "Vers l'atomisation du recours pour excès de pouvoir dans le contentieux des sanctions administratives », in RFDA, 2012, p.257.

MELLERAY, F., «Une nouvelle réduction du champ des mesures d'ordre intérieur en milieu carcéral», DA 2008, $n^{\circ} 2$, com $n^{\circ} 24$.

MOLINER-DUBOST, M., "A propos d'une autre « jurisprudence immobile ». Le contentieux des sanctions disciplinaires infligées aux détenus », in AJDA 2013, p.1380.

NIEVE, M., "Droit des détenus : sens et non-sens de la prison, un état des lieux du droit pénitentiaire en Belgique », Lette Actualités Droits-Libertés », in CREDOF, 26 mars 2013.

PASTOR, J-M., "Dignité du régime de détention et office du juge du référé provision », in AJDA, 2013, p2461.

PECHILLON, E., "L'accès au droit et le droit au recours », in CRDF 2004, $n^{\circ} 3$, p.49.

PONCELA, P., «Discipline pénitentiaire, un droit en mouvement », in RSC 2012, p.209.

PRADEL, J., "Les nouvelles règles pénitentiaires du Conseil de l'Europe», in RPDP, 1988, $n^{\circ} 2$, p. 218.

SAINT-PIERRE, F., et CORMIER, S., "L'avocat face à la nouvelle loi pénitentiaire », in RPDP, 2010, p.16.

SAUVE, J-M., "Le contrôle de l'administration pénitentiaire par le juge administratif », Discours à l'IEJ de Lille II, Conseil d'état, 24 février 2009.

VIGOUROUX, C., «La valeur de la justice en détention », in AJDA, 2009, p.403. 